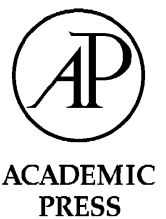

Available online at www.sciencedirect.com

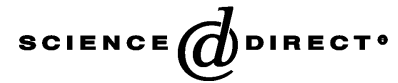

Journal of Sound and Vibration 271 (2004) 493-506

\title{
Experimental whole-field interferometry for transverse vibration of plates
}

\author{
Chien-Ching $\mathrm{Ma}^{\mathrm{a}, *}$, Chi-Hung Huang ${ }^{\mathrm{b}}$ \\ a Department of Mechanical Engineering, National Taiwan University, 1 Roosevelt Road, sec. 4, Taipei, Taiwan 106, ROC \\ ${ }^{\mathrm{b}}$ Department of Mechanical Engineering, Ching Yun Institute of Technology, Chung-Li, Taiwan 320, ROC
}

Received 30 January 2002; accepted 10 March 2003

\begin{abstract}
Most of the work on vibration analysis of plates published in the literature are analytical and numerical and very few experimental results are available. Existing modal analysis techniques such as accelerometers and laser Doppler vibrometers are pointwise measurement techniques and are used in conjunction with spectrum analyzers and modal analysis software to characterize the vibration behaviour. In this study, a whole-field technique called amplitude-fluctuation electronic speckle pattern interferometry optical system is employed to investigate the vibration behaviour of square isotropic plates with different boundary conditions. This method is very convenient to investigate vibration objects because no contact is required compared to classical modal analysis using accelerometers. High-quality interferometric fringes for mode shapes are produced instantly by a video recording system. Based on the fact that clear fringe patterns will appear only at resonant frequencies, both resonant frequencies and corresponding mode shapes can be obtained experimentally using the present method. Two different types of boundary conditions are investigated in this study, namely free-free-free-free (FFFF, 27 modes) and clamped-clamped-clampedclamped (CCCC, 12 modes). The numerical calculations by finite element method are also performed and the results are compared with the experimental measurements. Excellent agreements are obtained for both results of resonant frequencies and mode shapes.
\end{abstract}

(C) 2003 Elsevier Ltd. All rights reserved.

\section{Introduction}

Holographic interferometry is an optical method for full-field and non-contact measurement of object displacements. It opened new worlds of research by making global measurement of small dynamic surface displacements in a two-step process for a wide variety of objects. Unfortunately,

\footnotetext{
*Corresponding author. Tel.: + 8862-2365-9996; fax: + 886-2-2363-1755.

E-mail address: ccma@ntu.edu.tw (C.-C. Ma).
} 
holograms were recorded on photographic films and the cumbersome process of film development limits the application of holographic vibration analysis. Electronic speckle pattern interferometry (ESPI) was first proposed by Butters and Leendertz [1] as a method for producing the interferogram without using traditional film-based techniques. As compared with the traditional holographic interferometry [2], the interferometric fringe patterns of ESPI are recorded using video camera, which can eliminate the time-consuming chemical film development. Since the interferometric image is recorded and updated every $1 / 30 \mathrm{~s}$, ESPI is faster and more insensitive to environment than holography. For these reasons, ESPI has become a powerful technique used in many academic researches and engineering applications. Since ESPI used video recording and display, its real-time nature makes it practical for vibration measurement. ESPI can be applied to determine the resonant frequencies and to visualize the mode shapes of vibrating objects.

The most widely used experimental set-up to study dynamic responses by ESPI is the timeaveraged vibration ESPI method [3]. The disadvantage of this method is that the interferometric fringes represent the amplitude but not the phase of the vibration. To improve the shortcoming, the phase-modulation method that used the reference beam modulation technique was developed by Lokberg and Hogmoen [4] and Hurden [5] to determine the relative phase of the displacement. Shellabear and Tyrer [6] used ESPI to make three-dimensional vibration measurements. Three different illumination geometries were constructed, and the orthogonal components of vibration amplitude and mode shape were determined. For the purpose of reducing the noise coming from the environment, the subtraction method was developed by Creath et al. [7] and Pouet et al. [8]. The difference of the subtraction method compared with the time-averaged method is that the reference frame is first recorded before vibration and is continuously subtracted from the incoming frames after vibration. However, the interferometric fringe visibility of the subtraction method is not good enough for quantitative measurement of surface displacement. In order to increase the visibility of the fringe pattern and reduce environmental noise simultaneously, an amplitude-fluctuation ESPI (AF-ESPI) method was proposed by Wang et al. [9] for out-of-plane vibration measurement. In the AF-ESPI method, the reference frame is recorded in a vibrating state and subtracted from the incoming frame. Consequently, it combines the advantages of the time-averaged and subtraction methods, i.e., good visibility and noise reduction. Ma and Huang $[10,11]$ used the AF-ESPI method to investigate the three-dimensional vibrations of piezoelectric rectangular parallelepipeds and cylinders. Both the resonant frequencies and mode shapes were presented and discussed in details.

Thin plates have been extensively used as structural elements in many industrial applications. The investigation of the vibration of plates dates back to the 19th century. There had been a great amount of research and literature presented over the last century [12]. The problem of free vibration of a circular plate was first investigated by Poisson [13]. Rayleigh [14] presented the wellknown general method of solution to determine the resonant frequencies of vibrating structures. This method was improved by Ritz [15] by assuming a set of admissible trial functions. This approach is one of the most popular approximate methods for vibration analysis. There have been extensive studies of the vibration of plates for various shapes, boundary and loading conditions for nearly two centuries. The interested readers are referred to the excellent reviews of Leissa [16] and Liew et al. [17] of this class of problems and lists of references.

The study of the vibration behaviour of a plate is a problem of great practical interest and most of the published works are analytical and numerical results. There are very few experimental 
results available, especially for the full-field measurement of mode shapes. Model testing using pointwise measurement techniques (accelerometers or laser Doppler vibrometers) is commonly used in conjunction with spectrum analyzers and modal analysis software to characterize the vibration behaviour of plates. These pointwise techniques have suffered from irregular mode shape estimation as a result of the lengthy data acquisition period. In this paper, we employ an optical method based on the AF-ESPI to study experimentally the resonant characteristics (resonant frequencies and mode shapes) for isotropic square plates with different boundary conditions. The advantage of using the AF-ESPI method is that both resonant frequencies and the corresponding mode shapes can be obtained simultaneously. The fringe patterns shown in the experiment correspond to the vibrating mode shapes. Furthermore, it is very convenient to study small objects because no contact is required compared to classical modal analysis using accelerometers. Two cases are studied which involve the possible combinations of free and clamped edge conditions. They are free-free-free-free (FFFF) and clamped-clamped-clampedclamped (CCCC) plates. Because the proposed AF-ESPI optical method is faster in operation and insensitive to environment, a lot of excellent experimental vibration mode shapes are provided in this paper. There are 27 modes and 12 modes presented for FFFF and CCCC plates, respectively. The complete free (FFFF) case has a rich history in the vibration analysis literature. Ritz [15] analyzed the complete free problem to demonstrate the method provided by him for obtaining vibration frequencies. Due to the simplicity of the boundary condition, the completely clamped (CCCC) case is usually used as a test problem for analytical methods. In addition to the AF-ESPI experimental technique, numerical computations based on a finite element package are also presented, and good agreements are found in comparison with experimental results.

\section{AF-ESPI method for transverse vibration measurement}

The optical arrangement for out-of-plane vibrating measurement by AF-ESPI system is shown schematically in Fig. 1. If the image of the specimen is taken before vibration, the light intensity detected by a CCD camera can be expressed by time-averaged method as

$$
I_{0}=\frac{1}{\tau} \int_{0}^{\tau}\left(I_{A}+I_{B}+2 \sqrt{I_{A} I_{B}} \cos \phi\right) \mathrm{d} t=I_{A}+I_{B}+2 \sqrt{I_{A} I_{B}} \cos \phi,
$$

where $I_{A}$ is the object light intensity, $I_{B}$ the reference light intensity, $\tau$ the CCD refreshing time, and $\phi$ is the phase difference between object and reference light.

Assuming that the specimen vibrates periodically, the light intensity taken by a CCD camera can be represented as

$$
I_{1}=\frac{1}{\tau} \int_{0}^{\tau}\left\{I_{A}+I_{B}+2 \sqrt{I_{A} I_{B}} \cos \left[\phi+\frac{2 \pi}{\lambda}(1+\cos \theta) A \cos \omega t\right]\right\} \mathrm{d} t,
$$

where $\lambda$ is the wavelength of laser, $\theta$ the angle between object light and observation direction, $A$ the vibration amplitude, and $\omega$ is the angular frequency.

Let $\Gamma=2 \pi / \lambda(1+\cos \theta)$ and assume that the vibration period is much shorter than the CCD refresh time (i.e., $\tau \gg 2 \pi / \omega$ ). Then Eq. (2) can be worked out as

$$
I_{1}=I_{A}+I_{B}+2 \sqrt{I_{A} I_{B}}(\cos \phi) \mathrm{J}_{0}(\Gamma A),
$$




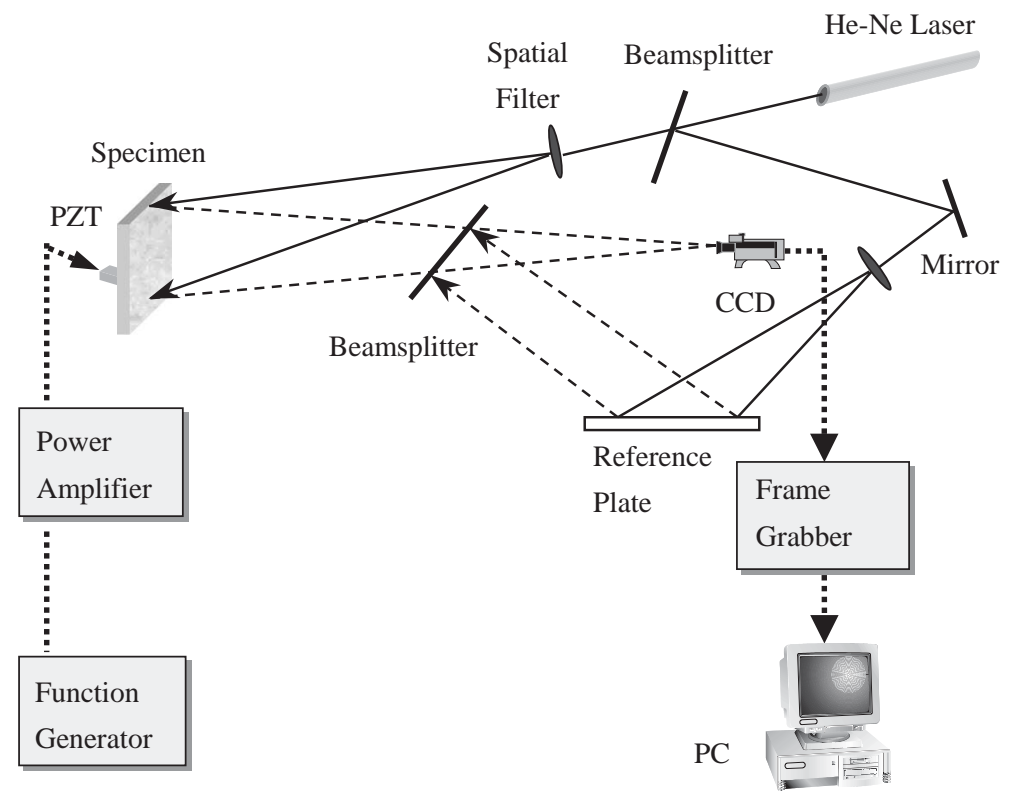

Fig. 1. Schematic layout of the experimental ESPI set-up for out-of-plane displacement measurement.

where $\mathrm{J}_{0}$ is a zero order Bessel function of the first kind. When these two images $\left(I_{0}\right.$ and $\left.I_{1}\right)$ are subtracted and rectified by the image processing system, i.e., subtract Eq. (1) from Eq. (3), the resulting image intensity can be expressed as

$$
I=I_{1}-I_{0}=2 \sqrt{I_{A} I_{B}}\left|(\cos \phi)\left[\mathrm{J}_{0}(\Gamma A)-1\right]\right| .
$$

The light intensity of the image shown in Eq. (4) is called the subtraction method, and the reference image is recorded before vibration.

Instead of using the subtraction method, the AF-ESPI method is employed in this study by taking two images while the specimen vibrates and assuming that the vibration amplitude of the second image has changed from $A$ to $A+\Delta A$. The light intensity of the second image will be

$$
I_{2}=\frac{1}{\tau} \int_{0}^{\tau}\left\{I_{A}+I_{B}+2 \sqrt{I_{A} I_{B}} \cos [\phi+\Gamma(A+\Delta A) \cos \omega t]\right\} \mathrm{d} t .
$$

Expanding Eq. (5) by using Taylor series and neglecting higher order terms one obtain

$$
I_{2}=I_{A}+I_{B}+2 \sqrt{I_{A} I_{B}}(\cos \phi)\left[1-\frac{1}{4} \Gamma^{2}(\Delta A)^{2}\right] \mathrm{J}_{0}(\Gamma A)
$$

When these two images $\left(I_{1}\right.$ and $\left.I_{2}\right)$ are subtracted and rectified by the image processing system, i.e., subtracting Eq. (3) from Eq. (6), the resulting image intensity can be expressed as

$$
I=I_{2}-I_{1}=\frac{\sqrt{I_{A} I_{B}}}{2}\left|(\cos \phi) \Gamma^{2}(\Delta A)^{2} \mathrm{~J}_{0}(\Gamma A)\right| .
$$

Compared with the subtraction method where the reference image is taken at stress-free state, the reference image is recorded and subtracted at the vibrating state by the AF-ESPI method. As indicated in Eqs. (4) and (7), the dominant function of the AF-ESPI method is $\left|\mathrm{J}_{0}(\Gamma A)\right|$ and that of the subtraction method is $\left|\mathrm{J}_{0}(\Gamma A)-1\right|$. Because of the discrepancy between the dominant 
functions, the nodal lines of vibrating interferometric patterns obtained by AF-ESPI method are the bright fringes and those by subtraction method are the dark fringes. This characteristic of AFESPI can be used for qualitative observation or for quantitative analysis for the fringe patterns. Furthermore, the sensitivity and fringe visibility of the AF-ESPI method are better than that of the subtraction method. It can be verified that the number of fringes presented by the AF-ESPI method is about twice that by the subtraction method for the same exciting force. In addition to the theory of out-of-plane measurement already mentioned, the in-plane vibration measurement by AF-ESPI method can also be derived in a similar way. By using the AF-ESPI method accompanied with the out-of-plane optical set-up, we can completely catch the full-field vibration characteristics of resonant frequencies and mode shapes at the same time.

\section{Experimental and numerical results}

Two isotropic aluminium thin plates (6061T6) are used in this study for experimental investigations and numerical calculations. The material properties of the plate are mass density $\rho=2700 \mathrm{~kg} / \mathrm{m}^{3}$, Young's modulus $E=70 \mathrm{GPa}$ and the Poisson ratio $v=0.33$. By using the combinations of free $(\mathrm{F})$ and clamped $(\mathrm{C})$ edges, the resonant frequencies and mode shapes of FFFF and CCCC plates are investigated. The geometric dimensions of two plates with different boundary conditions are shown in Fig. 2. The thickness of two isotropic plates is all $1 \mathrm{~mm}$.

The schematic layout of a self-arranged out-of-plane AF-ESPI optical system as shown in Fig. 1 is employed to perform the vibration measurement for the resonant frequency and the corresponding mode shape. As shown in Fig. 1, a continuous He-Ne laser (30 mW power) with wavelength $\lambda=632.8 \mathrm{~nm}$ is used as the coherent light source. The laser beam is divided into two parts, the reference and object beam, by a beamsplitter. We use a CCD camera (Pulnix company) and a P360F (Dipix Technologies Inc.) frame grabber with DSP on board to record and process the images. The object beam travels to the specimen and then reflects to the CCD camera. The reference beam goes directly to the CCD camera via a mirror and the reference plate. It is important to note that the optical path length and the light intensities of these two beams should remain identical in the experimental set-up. The CCD camera converts the intensity distribution of the interference pattern of the object into a corresponding video signal at 30 frames per second. The signal is electronically processed and finally converted into an image on the video monitor. The interpretation of the fringe image is similar to reading of a contour map. In order to increase the intensity of light reflection of specimens and the contrast of fringe patterns, the surfaces of these plates are coated with white paint, which is mixed with fine seaweed powder.

The isotropic plate is excited to resonance by a piezostack actuator (PI company), which is attached by adhesive behind the specimen. The piezoelectric actuator is usually attached in the centre of the specimen. However, if the nodal lines pass the centre of the specimen, then the piezoelectric actuator is moved to another location. The specimen is placed on a sponge for the FFFF case, the clamped sides of the CCCC plate are mounted with nine holes and are assumed to be fixed. To achieve the sinusoidal excitation input, a function generator HP33120A (Hewlett Packard) connected to a power amplifier (NF corporation) is used.

The experimental procedure of the AF-ESPI technique is performed as follows. First, a reference image is taken after the plate vibrates, then the second image is taken, and the reference 

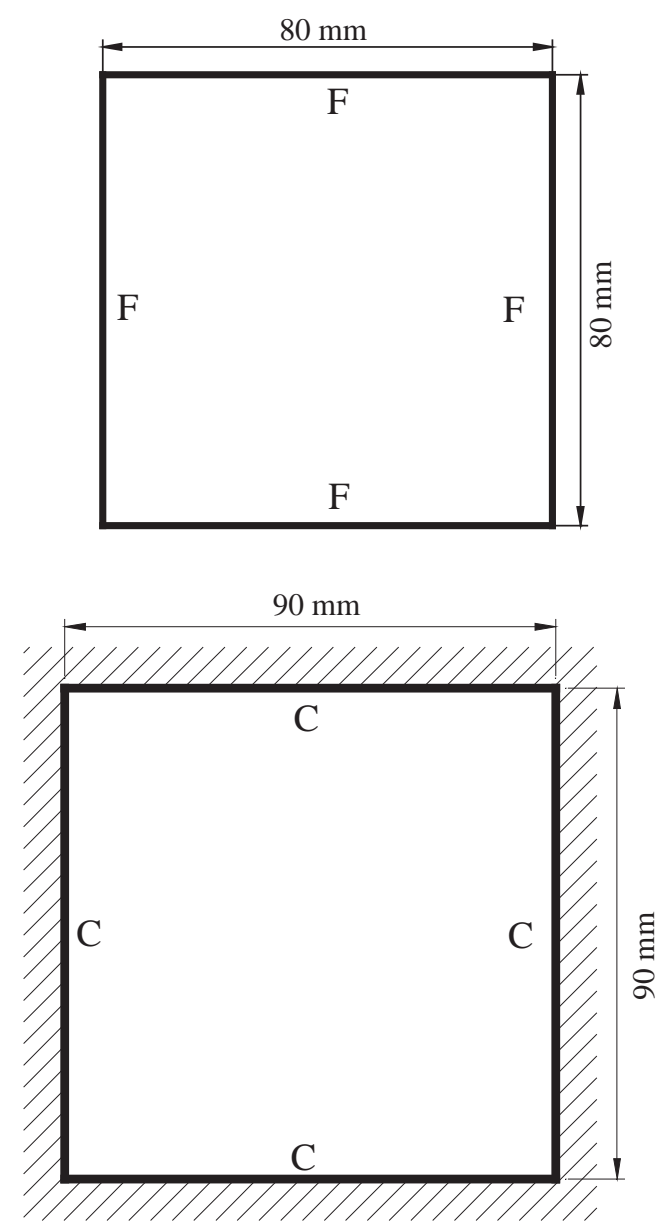

Fig. 2. Geometric dimension and configuration of isotropic square plates.

image is subtracted by the image processing system. If the vibrating frequency is not the resonant frequency, only randomly distributed speckles are displayed and no fringe patterns will be shown. However, if the vibrating frequency is in the neighbourhood of the resonant frequency, stationary distinct fringe patterns will be observed by the operator. Then the function generator is carefully and slowly turned; the number of fringes will increase and the fringe pattern will become clearer as the resonant frequency is approached. From the aforementioned experimental procedure, the resonant frequencies are recorded from the electronic counter and the corresponding mode shapes are observed by fringe patterns using the AF-ESPI optical system. The accuracy of the resonant frequency is given by the accuracy of the function generator and also by the skill of the user.

Numerical results of resonant frequencies and mode shapes are calculated by using the commercially available software, ABAQUS finite element package [18]. The eight-noded twodimensional quadrilateral thick shell element (S8R5) with $20 \times 20$ finite element meshes and reduced integration scheme are used to analyze the problem. This element approximates the 
Table 1

Comparison of theoretical predicted resonant frequencies with experimental results for the FFFF plate

\begin{tabular}{lllllllllllllll}
\hline Mode & 1 & 2 & 3 & 4 & 5 & 6 & 7 & 8 & 9 & 10 & 11 & 12 & 13 & 14 \\
\hline AF-ESPI (Hz) & 494 & 735 & 890 & 1271 & 2330 & 2345 & 2630 & 2930 & 3895 & 4402 & 4630 & 4920 & 5654 & 5934 \\
FEM (Hz) & 510 & 745 & 945 & 1324 & 2357 & 2424 & 2634 & 2974 & 4021 & 4499 & 4731 & 5048 & 5827 & 6163 \\
Error (\%) & 3.1 & 1.3 & 5.8 & 4.0 & 1.1 & 3.3 & 1.5 & 1.5 & 3.1 & 2.2 & 2.1 & 2.5 & 3.0 & 3.7 \\
& & & & & & & & & & & & & & \\
Mode & 15 & 16 & 17 & 18 & 19 & 20 & 21 & 22 & 23 & 24 & 25 & 26 & 27 \\
AF-ESPI (Hz) & 6294 & 7400 & 7550 & 8104 & 9290 & 10560 & 10790 & 10920 & 11100 & 11160 & 12440 & 12780 & 13400 \\
FEM (Hz) & 6458 & 7628 & 7810 & 8221 & 9296 & 10709 & 11125 & 11267 & 11342 & 11403 & 12857 & 13206 & 13858 \\
Error (\%) & 2.5 & 3.0 & 3.3 & 1.4 & 0.6 & 1.4 & 3.0 & 3.1 & 2.1 & 2.1 & 3.2 & 3.2 & 3.3 \\
\hline
\end{tabular}

Table 2

Comparison of theoretical predicted resonant frequencies with experimental results for the CCCC plate

\begin{tabular}{lllllllllllll}
\hline Mode & 1 & 2 & 3 & 4 & 5 & 6 & 7 & 8 & 9 & 10 & 11 & 12 \\
\hline AF-ESPI (Hz) & 1078 & 2181 & 3211 & 3904 & 3925 & 4872 & 6198 & 6485 & 7085 & 7190 & 8553 & 8964 \\
FEM (Hz) & 1099 & 2240 & 3300 & 4011 & 4030 & 5025 & 6408 & 6691 & 7362 & 7393 & 8997 & 9385 \\
Error (\%) & 1.9 & 2.6 & 2.7 & 2.7 & 2.6 & 3.0 & 3.3 & 3.1 & 3.8 & 2.7 & 4.9 & 4.5 \\
\hline
\end{tabular}

Midlin-type element that accounts for rotary inertia effects and first order shear deformations through the thickness.

The results presented in Tables 1 and 2 show generally good agreement between the numerically predicted and experimentally measured resonant frequencies. The resonant frequencies ranged from 494 to $13,400 \mathrm{~Hz}$ for the FFFF plate and ranged from 1078 to $8964 \mathrm{~Hz}$ for the CCCC plate. The error in resonant frequency prediction is given by

$$
\% \text { Error }=\frac{f_{\text {theory }}-f_{\text {exp }}}{f_{\text {theory }}} 100 \% .
$$

The percentage differences between the experimental data and the analytical results are also shown in Tables 1 and 2 . The worst error is $5.8 \%$ and the average error in the results is $2.8 \%$. All the experimental measured frequencies are lower than the numerical ones. The errors are probably due to thickness variations across the plates, the material property measurement, the weight of the PZT actuator, the finite element approximation method and the clamped boundary condition of the plate.

The resonant frequency $f$ is also expressed in terms of a non-dimensional frequency parameter $\lambda^{2}$ given by

$$
\lambda^{2}=2 \pi f L^{2} \sqrt{\frac{12 \rho\left(1-v^{2}\right)}{E h^{2}},}
$$

where $L$ is the length and $h$ is the thickness of the square plate. Figs. 3 and 4 show the relation of non-dimensional resonant frequency and mode number for square plates with different boundary 


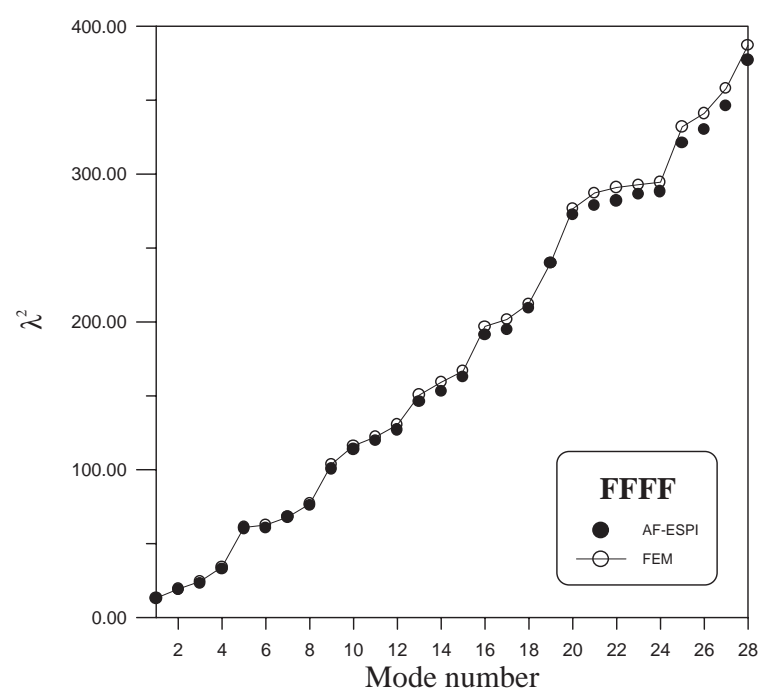

Fig. 3. Comparison of the non-dimensional frequencies by AF-ESPI and FEM for the FFFF plate.

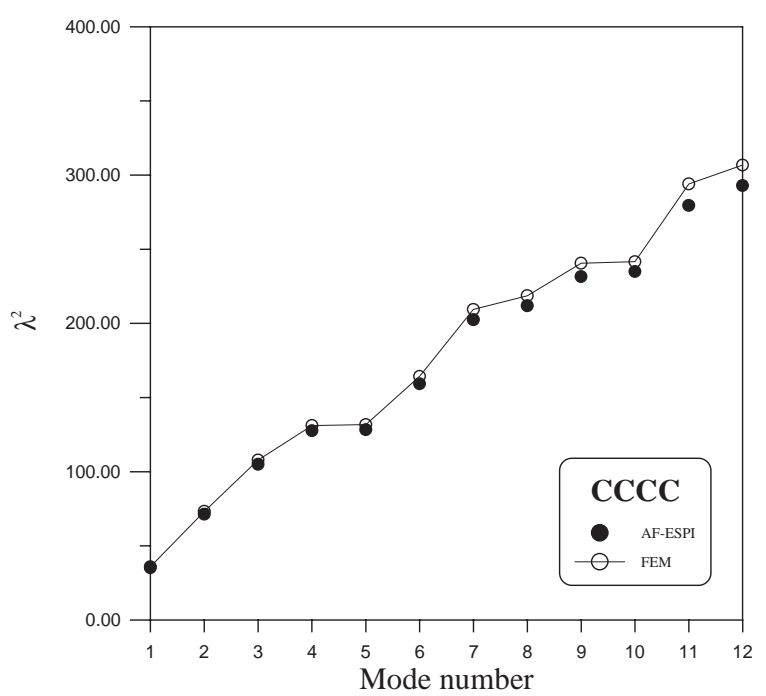

Fig. 4. Comparison of the non-dimensional frequencies by AF-ESPI and FEM for the CCCC plate.

conditions. Since the weight of the specimen increases slightly by the attached actuator and the clamped edge in experiment is not as rigid as the theoretical assumption, the resonant frequencies obtained from experimental measurement turn out to be lower than the numerical results.

Figs. 5 and 6 are the mode shapes for both experimental measurements and numerical simulations. There are 27 modes and 12 modes presented for FFFF and CCCC plates, respectively. For the finite element calculations, the contours of constant displacement for resonant mode shapes are plotted in order to compare with the experimental observation. In Figs. 5 and 6, we indicate the phase of displacement in finite element results as solid or dashed 

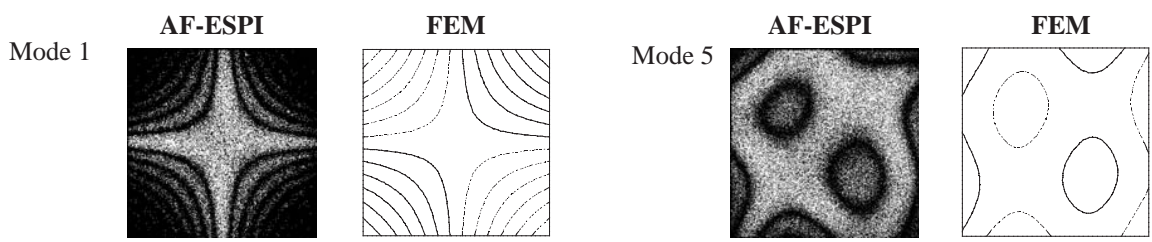

Mode 2
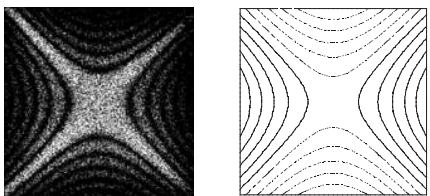

Mode 6
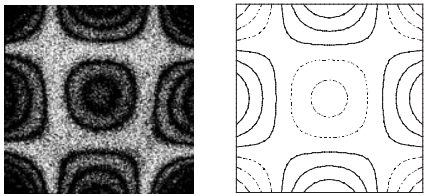

Mode 3
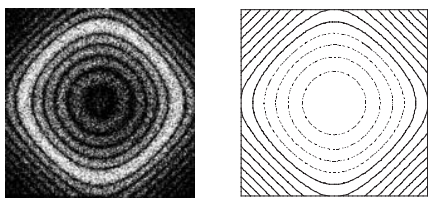

Mode 7
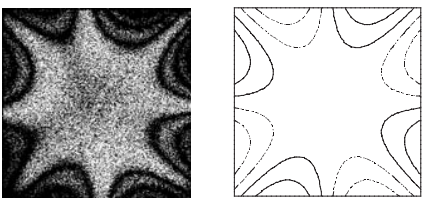

Mode 4
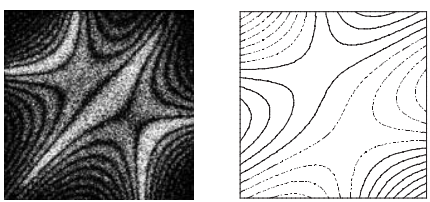

Mode 8
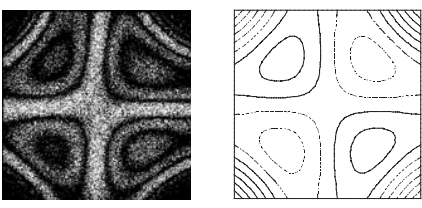

Mode 9
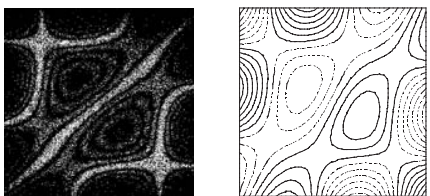

Mode 13
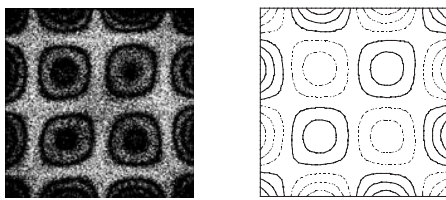

Mode 10
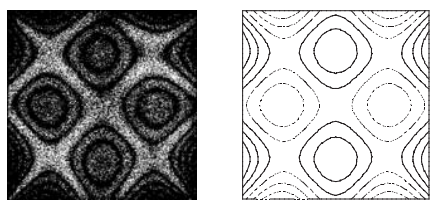

Mode 14
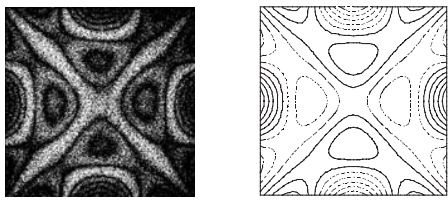

Mode 11
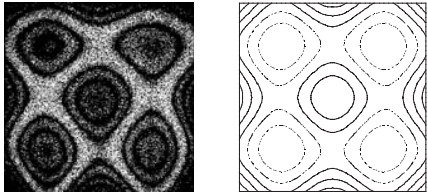

Mode 15
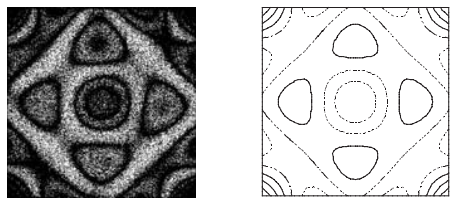

Mode 12
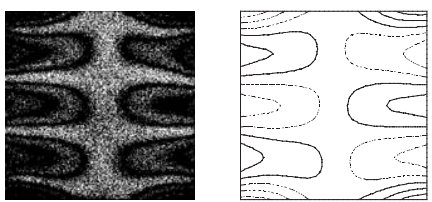

Mode 16
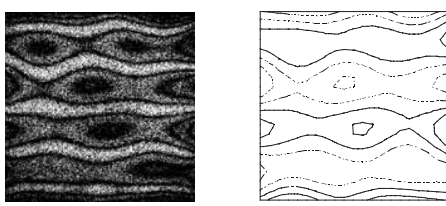

Fig. 5. The first 27 mode shapes obtained by using the experimental AF-ESPI system and the finite element analysis for the FFFF plate. 


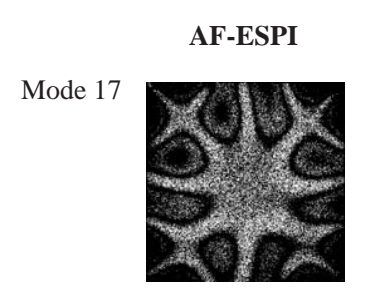

Mode 18

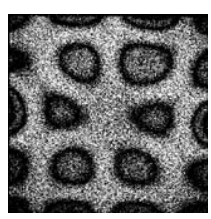

Mode 19

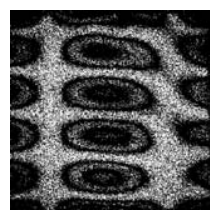

Mode 20

(e)

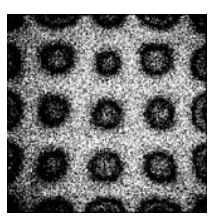

FEM
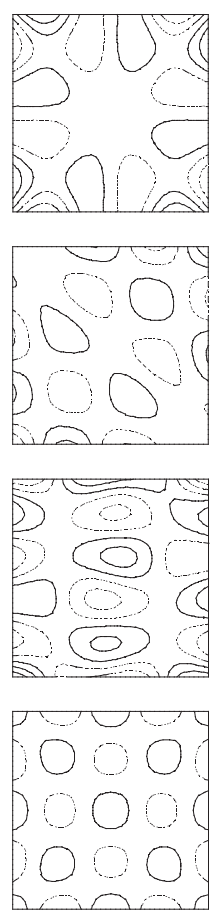

AF-ESPI

Mode 21

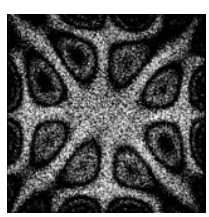

Mode 22

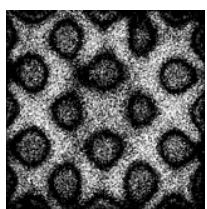

Mode 23

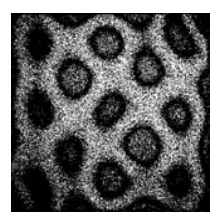

Mode 24

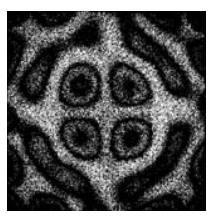

FEM

Mode 25

AF-ESPI

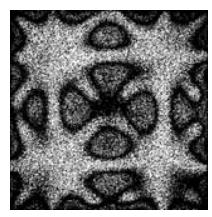

Mode 26

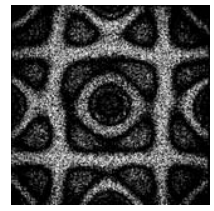

Mode 27

(g)

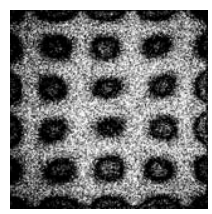

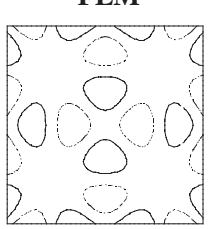
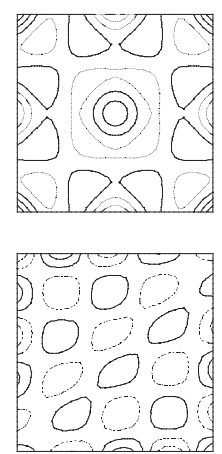

Fig. 5 (continued).

line, the solid lines are in the opposite direction to the dashed lines. The transition from solid lines to dashed lines corresponds to a zero displacement line, or nodal line. The zero order fringe, which is the brightest on the experimental image represents the nodal lines of the vibrating square plate 

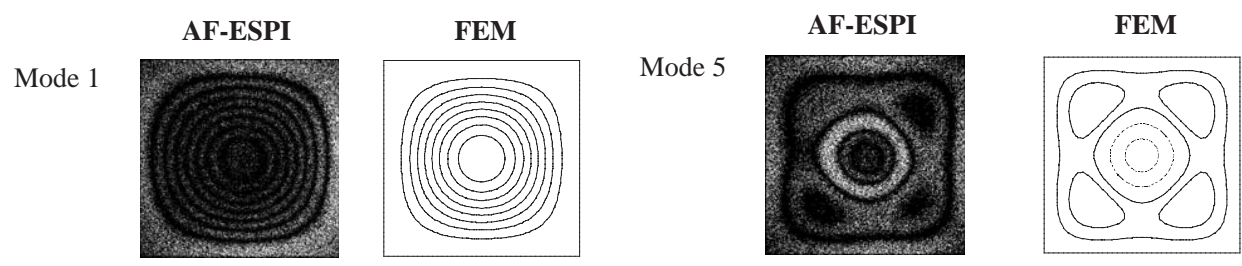

Mode 2
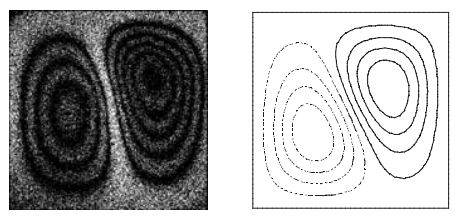

Mode 6
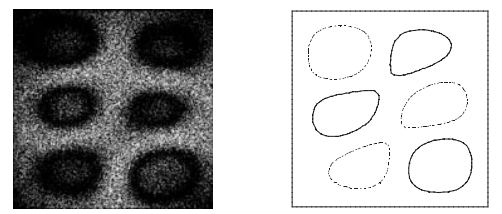

Mode 3
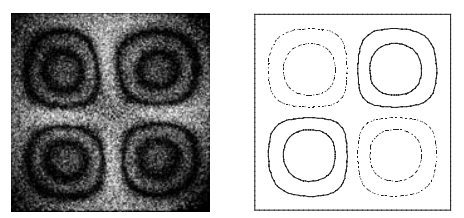

Mode 7
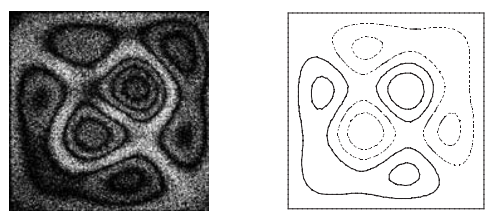

Mode 4
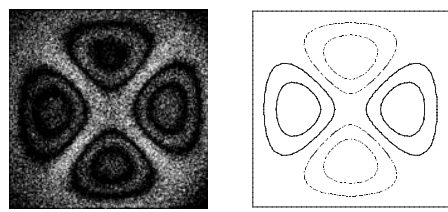

Mode 8
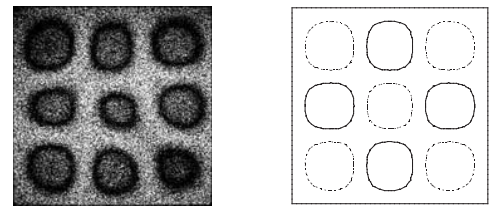

(b)

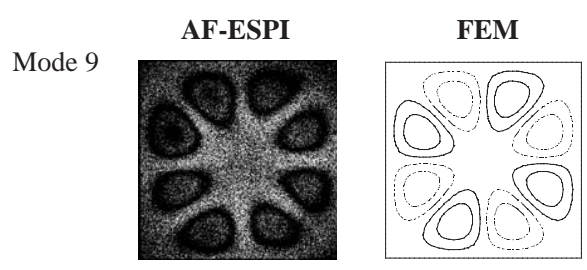

Mode 10
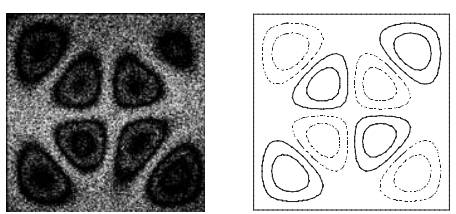

Mode 11
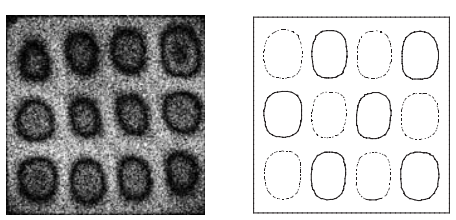

Mode 12
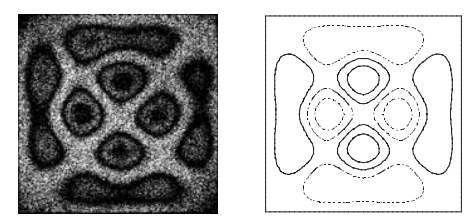

Fig. 6. The first 12 mode shapes obtained by using the experimental AF-ESPI system and the finite element analysis for the CCCC plate. 
at resonant frequencies. The rest of the fringes are contours of constant amplitudes of displacement. Excellent quality of the experimental fringe patterns for vibration mode shapes are presented in Figs. 5 and 6 . The mode shapes obtained by experimental results can be checked by the nodal lines and fringe patterns with the numerical finite element calculations and excellent agreements are found.

Due to the symmetry of the geometric configuration of the isotropic square plate and the boundary condition, there may be two possible mode shapes at the same resonant frequency. If the plate is excited at this frequency, a linear combination of these modes will be obtained in experimental observation and it is not possible to define uniquely the mode shape of the vibration. The precise nature of the mode produced in experiment depending on the position and method of excitation. There are a few examples for this case, for instance modes 4, 5, 9, 12, 16, etc., in the FFFF plate. A detailed discussion of this phenomenon was provided by Leissa [12].

It is noted that, for high-frequency modes, there may be cases of mode skipping and switching. A comparison of the resonant frequencies of composite laminated plates obtained by using the classical plate theory and the third order shear deformation theory were presented by Hadian and Nayfeh [19]. They found that, as the mode number increases, the resonant frequency obtained by using the classical plate theory deviates from that obtained by the high order shear deformation theory. The switching for high-frequency modes was observed experimentally by Oh an Nayfeh [20] for investigation the vibration of a cantilever six-layer laminated graphite-epoxy composite plate. They explained the switching in the high order modes may be due to neglecting higher order shear deformations. However, we do not find any mode switching or skipping in this study, the sequence of all the modes calculated from the finite element simulation is the same as that obtained by the experimental observation.

\section{Conclusions}

Optical techniques have been shown to have certain advantages for vibration analysis and ESPI has been applied to many vibration problems. The advantages of the optical ESPI method include the full-field measurement, real-time observation, submicron sensitivity, validity of both static deformation and dynamic vibration, and direct digital image output. Moreover, no equipment, transducer or sensor, needs to be attached to the plate in order to record the vibration. This method can be applied to many cases within a range of displacements between tens of nanometers and tens of micromters. Because ESPI uses video recording and display, it works in real time to measure dynamic displacement, which enables implementation of this technique for vibration measurement.

The vibration behaviour of isotropic plates are well-understood theoretically with computational results and data stretching back many years. However, there are only very few experimental results available in the literature, especially for the full-field measurement of vibration mode shapes. If the plate is thin, then it displays large transverse (out-of-plane) motion compared to any possible in-plane components. A self-arranged AF-ESPI optical set-up with good fringe visibility and noise reduction has been used in this study to obtain the resonant frequencies and the corresponding mode shapes of free vibration of isotropic square plates at the same time. Compared with the spectrum analysis or modal analysis method, AF-ESPI is more convenient in 
experimental operation. Two different types of boundary conditions are investigated in this study and about forty excellent quality of mode shapes are generated using the experimental optical interferometry method. Numerical calculations of resonant frequencies and mode shapes based on a finite element package are also performed and excellent agreements are obtained when compared with experimental measurements. It is noted that experimental frequencies obtained are typically lower than theoretical ones because one cannot get perfectly rigid clamping experimentally. The results shown in this study demonstrate that the AF-ESPI method is applicable to many situations in engineering vibration analysis as long as the vibration amplitude reaches the sensitivity of AF-ESPI method.

\section{Acknowledgements}

The authors gratefully acknowledge the financial support of this research by the National Science Council (Republic of China) under Grant NSC 88-2212-E002-046.

\section{References}

[1] J.N. Butters, J.A. Leendertz, Speckle pattern and holographic techniques in engineering metrology, Optics and Laser Technology 3 (1971) 26-30.

[2] P.K. Rastogi, Holographic Interferometry, Springer, Berlin, 1994.

[3] R. Jones, C. Wykes, Holographic and Speckle Interferometry, Cambridge University Press, Cambridge, 1989.

[4] O.J. Løkberg, K. Hogmoen, Use of modulated reference wave in electronic speckle pattern interferometry, Journal of Physics E: Scientific Instruments 9 (1976) 847-851.

[5] A.P.M. Hurden, An instrument for vibration mode analysis using electronic speckle pattern interferometry, NDT International 15 (1982) 143-148.

[6] M.C. Shellabear, J.R. Tyrer, Application of ESPI to three-dimensional vibration measurements, Optics and Lasers in Engineering 15 (1991) 43-56.

[7] K. Creath, G.A. Slettemoen, Vibration-observation techniques for digital speckle-pattern interferometry, Journal of the Optical Society of America A 2 (1985) 1629-1636.

[8] B. Pouet, T. Chatters, S. Krishnaswamy, Synchronized reference updating technique for electronic speckle interferometry, Journal of Nondestructive Evaluation 12 (1993) 133-138.

[9] W.C. Wang, C.H. Hwang, S.Y. Lin, Vibration measurement by the time-averaged electronic speckle pattern interferometry, Applied Optics 35 (1996) 4502-4509.

[10] C.H. Huang, C.C. Ma, Vibration characteristics for piezoelectric cylinders using amplitude-fluctuation electronic speckle pattern interferometry, American Institute of Aeronautics and Astronautics Journal 36 (1998) 2262-2268.

[11] C.C. Ma, C.H. Huang, The investigation of three-dimensional vibration for piezoelectric rectangular parallelepipeds by using the AF-ESPI method, IEEE Transactions on Ultrasonics Ferroelectrics and Frequency Control 48 (2001) 142-153.

[12] A.W. Leissa, Vibration of Plates, NASA SP-160, US Government Printing Offices, Washington, DC, 1969.

[13] S.D. Poisson, L'Equilibre et le mouvement des corps elastiques, Memoires de L'Academie Royale des Sciences de L'Institute de France 8 (1829) 357-570.

[14] L. Rayleigh, Theory of Sound, Vol. 1, Macmillan, London (reprinted 1945 by Dover, New York).

[15] W. Ritz, Ueber eine neue methode zur loesung gewisser variations probleme der mathematischen physic, Journal fuer Reine und Angewandte Mathematick 135 (1909) 1-61.

[16] A.W. Leissa, Recent studies in plate vibration: 1981-85, Part 1, classical theory, The Shock and Vibration Digest 19 (1987) 11-18. 
[17] K.M. Liew, Y. Xiang, S. Kitipornchai, Research on thick plate vibration: a literature survey, Journal of Sound and Vibration 180 (1995) 163-176.

[18] Abaqus User's Manual, Version 5.5, Hibbitt, Karlsson and Sorensen, Inc., Rhode Island, 1995

[19] J. Hadian, A.H. Nayfeh, Free vibration and buckling of shear-deformable cross-ply laminated plates using the step-space concept, Computers and Structures 48 (1993) 677-693.

[20] K. OH, A.H. Nayfeh, High-to-low frequency modal interactions in a cantilever composite plate, American Society of Mechanical Engineers, Journal of Vibration and Acoustics 120 (1998) 579-587. 